\section{Bile acid-microbiota crosstalk in gastrointestinal inflammation and carcinogenesis: a role for bifidobacteria and lactobacilli?}

\section{Borja Sánchez}

In a recent excellent Review ${ }^{1}$ (Bile acidmicrobiota crosstalk in gastrointestinal inflammation and carcinogenesis. Nat. Rev. Gastroenterol. Hepatol. 15, 111-128 (2018)), the connection between human and microbial bile acid (BA) metabolism was described. In this Review, the authors identified possible therapeutic targets from data published in the context of gastrointestinal inflammation and carcinogenesis. From the microbial point of view, BAs are one of the main factors determining probiotic survival through the human gastrointestinal tract, which indeed was the focus of my $\mathrm{PhD}$ work ${ }^{2}$.

In this regard, I feel that one important process as part of the interaction between BAs and probiotic bacteria is missing in the global picture presented in this Review: at least two of the main probiotic representatives, bifidobacteria and lactobacilli, accumulate primary unconjugated BAs (such as cholic acid (CA)) and probably secondary unconjugated BAs (such as deoxycholic acid (DCA)) in their cytoplasm either spontaneously, or following intracellular BA deconjugation ${ }^{3,4}$. In the latter case, BAs are generated after the action of the bile salt hydrolase (BSH), a cytoplasmic enzyme catalysing the hydrolysis of a conjugated BA into its unconjugated and amino acid moieties. Unconjugated BA accumulation requires cell energization, reflecting the situation happening during transient probiotic growth in the gut. BAs are weak acids that are transported in their neutral form (protonated) into the bacterial cytoplasm, where they quickly dissociate, losing a proton and lowering the internal $\mathrm{pH}$ of the bacterium ${ }^{5}$. Indeed, the mechanisms of resistance to BAs and low $\mathrm{pH}$ values are connected in the Bifidobacterium lactis species through the membrane-bound $\mathrm{F}_{1}-\mathrm{F}_{0}$ ATPase; the high activity of this enzyme compared with other bifidobacteria together with its intrinsic aerotolerance explains why $B$. lactis is predominant in probiotic foods containing live bifidobacteria ${ }^{6}$.

Secondary BAs are produced from primary BAs by the action of the human gut microbiota $^{7}$. For instance, CA is dehydroxylated at the $\mathrm{C} 7$ position by the enzymatic action of strains belonging to the genera Clostridium and Eubacterium, resulting in the formation of $\mathrm{DCA}^{8}$. As pointed out by the authors ${ }^{1}, \mathrm{DCA}$ is involved in the progression of colorectal cancer, notably in the context of obesity, which is also characterized by an ongoing low-grade inflammatory status. Gut concentration of pro-inflammatory and pro-carcinogenic DCA is therefore directly related to the composition of the intestinal microbiota, but the levels of this secondary BA might be modified by probiotic intake or by the presence of higher levels of resident bifidobacteria and/or lactobacilli. The hypothesis is that primary BAs sequestered in the cytoplasm of these bacteria will escape the portal circulation and the action of other microorganisms to produce secondary BAs. Non-converted primary BAs would be eliminated with the faeces.

BA accumulation in bifidobacteria and lactobacilli, with many strains being considered as probiotics and others representatives of the human gut microbiota, might have implications in chronic inflammation and carcinogenesis through this BA-accumulation mechanism. For that reason, higher levels of these bacteria (and perhaps other BSHcontaining Gram-positive bacteria) or regular probiotic intake could be an interesting means of reducing colonic DCA levels and possibly decreasing inflammation and colorectal cancer risk. In this sense, experimental data support the probiotic ability to reduce DCA levels in vitro 9 . This aspect is of paramount importance as probiotics (lactobacilli and bifidobacteria) might be used as a means of decreasing DCA levels through deconjugation of conjugated CA or DCA or accumulation of primary or secondary BAs, with both cases resulting in the sequestration of those BAs in the large intestine.

Borja Sánchez

Dairy Research Institute of Asturias, Spanish National Research Council (IPLA-CSIC), Asturias, Spain. e-mail: borja.sanchez@csic.es doi: $10.1038 /$ nrgastro.2018.23 Published online 7 Mar 2018

1. Jia, W., Xie, G. \& Jia, W. Bile acid-microbiota crosstalk in gastrointestinal inflammation and carcinogenesis. Nat. Rev. Gastroenterol. Hepatol. 15, 111-128 (2018).

2. Ruiz, L., Margolles, A. \& Sánchez, B. Bile resistance mechanisms in Lactobacillus and Bifidobacterium. Front. Microbiol. 4, 396 (2013).

3. Kurdi, P. et al. Cholic acid is accumulated spontaneously, driven by membrane $\Delta \mathrm{pH}$, in many lactobacilli. J. Bacteriol. 182, 6525-6528 (2000).

4. Kurdi, P. et al. Cholic acid accumulation and its diminution by short-chain fatty acids in bifidobacteria. Microbiology 149, 2031-2037 (2003).

5. Kurdi, P. et al. Mechanism of growth inhibition by free bile acids in lactobacilli and bifidobacteria mechanism of growth inhibition by free bile acids in lactobacilli and bifidobacteria. J. Bacteriol. 188, 1979-1986 (2006).

6. Sánchez, B., de los Reyes-Gavilán, C. G. \& Margolles, A The F1F0-ATPase of Bifidobacterium animalis is involved in bile tolerance. Environ. Microbiol. 8, 1825-1833 (2006).

7. Ridlon, J. M., Kang, D.-J. \& Hylemon, P. B. Bile salt biotransformations by human intestinal bacteria. J. Lipid Res. 47, 241-259 (2006).

8. Wells, J. E. \& Hylemon, P. B. Identification and characterization of a bile acid $7 \alpha$-dehydroxylation operon in Clostridium sp. strain TO-931, a highly active $7 \alpha$-dehydroxylating strain isolated from human feces. Appl. Environ. Microbiol. 66, 1107-1113 (2000).

9. Guo, C. F. et al. Screening for cholesterol-lowering probiotic based on deoxycholic acid removal pathway and studying its functional mechanisms in vitro. Anaerobe 18, 516-522 (2012).

Competing interests

B.S. is a scientific founder of Microviable Therapeutics. 